\title{
SPECTROSCOPIC CRITERIA FOR THE CLASSIFICATION OF \\ PECULIAR B-TYPE STARS WITH \\ COLOUR-SPECTRUM DISCREPANCIES
}

\author{
R. F. GARRISON \\ David Dunlap Observatory, University of Toronto, Richmond Hill, Ontario, Canada
}

\begin{abstract}
The so-called 'weak helium' stars are discussed from a spectral classification point of view. Several authors have included different kinds of objects in this class. Often, the only similarity is the discrepancy between the spectra and the colours. It is desirable to decouple the discovery of new members of the class from this dependence on colours so that the colour can be used as an independent parameter.
\end{abstract}

I would like to use this opportunity to make some comments on the technique and philosophy of spectral classification, then give an example and a model. As the example, I have chosen the peculiar B stars with colour-spectrum discrepancies.

The first point concerns the relationship between photometry and spectral classification. It is assumed by many astronomers that there is a one-to-one relationship between the two and that photometry can replace classification or vice-versa. In my experience this is not so, and the best use of the two techniques is in taking advantage of their complementarity.

The second point concerns the relationship between high dispersion and low dispersion. It is usually assumed that if something can be done well with low dispersion spectra, it can be done that much better with higher resolution. In my experience, this is not always the case, because of the greater visibility of wings and blends with low dispersion as well as the ease of looking at the spectrum as a whole. Thus, high dispersion and low dispersion studies are also complementary rather than competitive.

The example of the so-called 'weak helium' peculiar B stars is a good illustration of the complementarity of these techniques. This is a class of stars which were picked out as spectroscopically peculiar, without reference to their colours, during a study of the members of the association in upper Scorpius (Garrison, 1967, see notes to Table III).

A superficial classification would assign them to the region of the B7-9 giants, but a closer examination would reveal several peculiarities which can be detected at MK dispersion.

(1) Broad, washed out $\mathrm{H}$ wings and $\mathrm{He}$ lines (by 'washed out', I mean apparently filled in by continuum emission).

(2) Cores of $\mathrm{H}$ are normal for either B8III or B3V. This with (1), gives the $\mathrm{H}$ lines a characteristic and easily recognized shape at low dispersion which is not apparent in high dispersion spectra.

(3) The presence, usually but not always, of C II and Si III lines, which are characteristic of B3 stars. 
(4) The presence, occasionally, of faint lines of Fe II and Ti II, which are characteristic of A and late B-type stars.

When the MK type, say B8IIIp, (the $p$ indicating the above characteristics) is compared with the colours, it is invariably found that the colours are those of a B3-5 star. This discrepancy is clearly shown by plotting $(U-B)_{0}$ vs MK type (Garrison, 1967, Figure 7). Such colour-spectrum discrepancies were noted earlier by Sharpless (1952), Jugaku and Sargent (1961), and McNamara and Larsson (1962). Later, the discrepancy was used to pick out such stars; e.g. Bernacca (1968), Jaschek et al. (1969). (Note that Table IV in the latter paper contains a large number of errors, which seem to have eluded both authors and editors.) However, it is necessary to use spectroscopic criteria to distinguish the 'weak helium' stars from stars of a rather different peculiar nature, e.g. well known Ap types, such as $\mathrm{Si}, \mathrm{Hg}, \mathrm{Mn}$, etc., and the uniquely peculiar object 36 Lyn, which does not resemble the rest of the group.

There are several reasons for decoupling the colour from the spectrum, in discovery surveys at least. It may well be that these stars will turn out to be hot analogues of the Ap stars but it is desirable to know their colour characteristics as distinct from their spectral characteristics; therefore the relationship between colour and spectrum should not be assumed a priori. Secondly, it may well be that some 'normal' stars exhibit some of the same characteristics without the colour discrepancy. If the colour and spectrum were coupled a priori, then interesting examples would be missed. Thirdly, there is some indication that the presence of Fe II may be correlated with colour (Jaschek, 1971), but a pure sample, unrelated to colour, would be necessary to establish this.

This leads to a consideration of a model for Bp stars. The obvious model is that of a B3V star with helium underabundant by a factor of 100 , or more. There are several objections to this interpretation, none of which alone is sufficient, but the weight of all of them makes the interpretation unsatisfactory.

(1) Many of these stars are physical members of young clusters and associations (Orion, $\alpha$ Persei, Sco-Cen, etc.) and coexist with stars of 'normal' helium abundance or even 'helium rich' stars (in the case of the $\sigma$ Ori subgroup).

(2) The peculiar spectrum variable a Cen resembles a 'helium poor' star at one phase and a 'helium rich' star at another. It varies back and forth with a period of slightly more than one week. During this period there are differential velocity variations and spectral changes which indicate extreme density variations (Klinglesmith, Bernacca, and Frey, 1971). Figure 1 is an illustration of the variations in this remarkable star.

(3) The spectra at low dispersion have many of the characteristics of very mild shell stars and the variations in a Cen, described in (2), indicate non-LTE effects.

(4) When plotted according to their colours in well-determined HR diagrams, such as $\alpha$ Persei (Morgan, Hiltner, and Garrison, 1971), the Orion Belt, and Upper Scorpius (Garrison, 1967), they fall 0.5-0.75 mag. below the main sequence.

(5) The presence of faint lines of Ti II and Fe II in some spectra suggests that if B3 


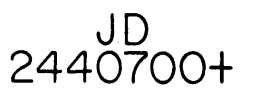

63.67

64.45

65.46

66.53

67.46

68.45

70.45

71.66

73.45

74.71

75.47

76.46

77.45

78.47

79.46

79.67

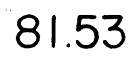

HD 125823 (a Cen)

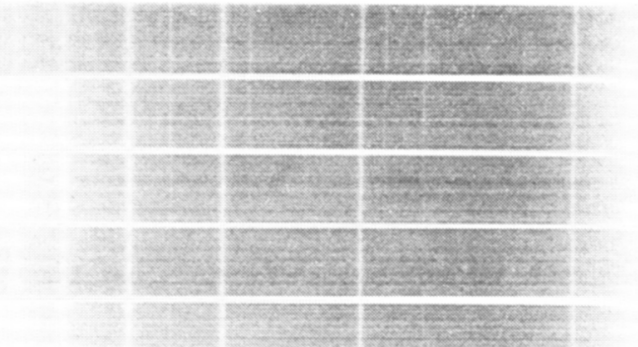

26 JUNE

27

28

29

30
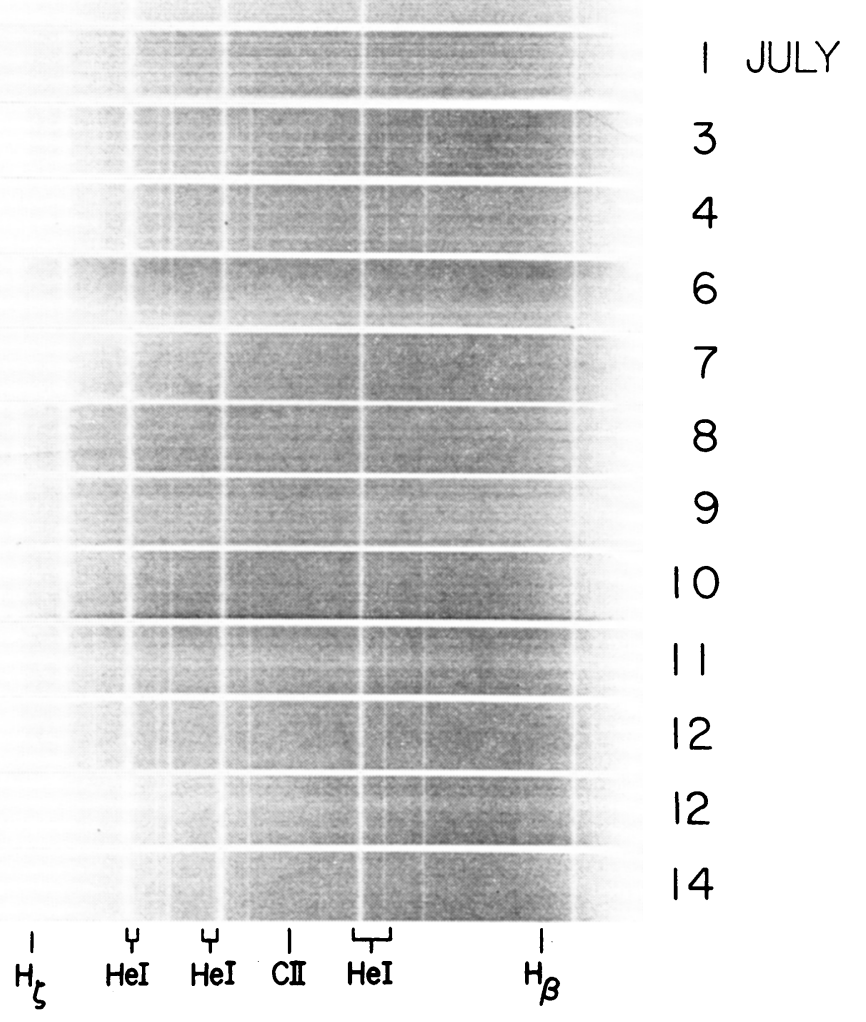

1970 UT

Fig. 1. Cerro Tololo spectrograms of a Cen (HD 125823). Original dispersion $91 \AA^{-m_{m}^{-1}}$ at $\mathbf{H} \gamma$. Note the extreme changes in $\mathrm{He}$ I strength. Variations are observed in $\mathrm{C}$ II and possibly in $\mathrm{H}$. The colour is that of a B2 star and changes very little during the cycle. At maximum He strength, the spectrum resembles the 'helium rich' stars and at minimum, the 'weak helium' group.

is assumed, then not only is $\mathrm{He}$ underabundant, but $\mathrm{Fe}$ and $\mathrm{Ti}$ are overabundant. This involves too many abundance anomalies.

The model which I would like to propose here, to stimulate discussion, is one in which the underlying star is B3-5 but with a semi-opaque electron-scattering shell located within or near the photosphere; in other words, large departures from the 
normal density-radius relation. This provides for the spectroscopic characteristics and explains the spectrum-colour discrepancy. Details have not been worked out quantitatively.

More observations are obviously needed for these stars. In particular, statistical studies and high dispersion studies of line identifications, line profiles, and velocities would be useful.

High dispersion studies are being undertaken by several groups. Norris, now at Yale, is studying HD 142301 in Upper Scorpius. One of my students, Bruckner, is studying HD 21699 in the $\alpha$ Persei cluster and another, Campbell, is studying a group of 5 stars in the Orion Belt region. Bernacca, Klinglesmith, Molnar, Underhill and others at NASA are studying a Cen and a number of other members of the group.

The statistical studies are very difficult and time consuming if done properly. What percentage of the stars with B3-5 colours have B7-9 spectral characteristics? How many are in clusters and how many are in the field? In order to answer these and other questions properly, it is necessary to take all the HD stars from B3 to A0 to some limiting magnitude at MK dispersion for classification. This region of the HR diagram is often neglected, because the OB-type stars are more interesting for galactic structure and the A-type stars are more interesting for their spectacular peculiarities. Such a survey, without reference to colours, is being undertaken at the University of Toronto and will cover both northern and southern hemispheres, beginning with the bright stars.

From the preliminary results, the indications are that up to $20 \%$ of the stars with B3-5 colours have these peculiar characteristics. The observation that their luminosities are systematically lower than 'normal' stars with similar colours indicates that photometry needs to be supplemented by MK spectral classification if one is to obtain accurate distance moduli.

\section{Acknowledgements}

This work is being supported by the National Research Council of Canada.

I am also indebted to the Director and staff of the Cerro Tololo Inter-American Observatory for telescope time and to Chile for the excellent weather (even in winter) which enabled me to obtain the series on HD 125823 illustrated in Figure 1.

\section{References}

Bernacca, P. L.: 1968, Contr. Oss. Astrofiz. Univ. Padova, No. 202.

Garrison, R. F.: 1967, Astrophys. J. 147, 1003.

Jaschek, M., Jaschek, C., and Arnal, M.: 1969, Publ. Astron Soc. Pacific 81, 650.

Jaschek, M.: 1971, Private communication.

Jugaku, J. and Sargent, W. L. W.: 1961, Publ. Astron. Soc. Pacific 73, 249.

Klinglesmith, D. A., Bernacca, P. L., and Frey, H.: 1971, Bamberg Conference on Variable Stars,

Veroeffentl. Remeis-Sternwarte Bamberg 9, Nr. 100, p. 205.

McNamara, D. H. and Larsson, H. J.: 1962, Astrophys. J. 135, 748.

Morgan, W. W. Hiltner, W. A., and Garrison, R. F.: 1971, Astrophys. J. 76, 242.

Sharpless, S.: 1952, Astrophys. J. 116, 251 